\section{Differentiated Psychopathology and Molecular Genetics of Endogenous Psychosis: Much More than a Misunderstanding}

Received: November 26, 2015; Accepted: November 27, 2015, Published: December 10, 2015

The search for biological basis of psychiatric disorders like schizophrenia, means a big endeavour that cope with a myriad of complex issues: Brain structure, chemistry, connectivity, genetics and development; environmental normal and noxious stimuli including early childbearing and trauma; Effects of drugs of abuse and modern lifestyles and threatens; manifold psychopathological manifestations of every condition, including the way they change our mode of being in the world; Complex environmental/genetic interactions; Effect of psychotropic and psychotherapeutic interventions. This is only a sketch of the universe we are dealing with. But modern psychiatry has been tempted several times to make simpler the complex in attempts to gain knowledge. Psychopathology of endogenous psychosis, and its complex relationships with biology, particularly genetics, is a good example.

Last edition of the Diagnostic and Statistical Manual of Mental Disorders (DSM 5) (American Psychiatric Association, 2013) [1] shows a big change in the chapter of schizophrenias: classical clinical forms have been eliminated in favour of a "dimensional" approach. A complete new framework supported more by good ideas a la page than by empirical data are aimed to replace 120 years of specialist's wisdom. This argument seems far from being a solid scientific decision. It is true that DSM subclinical forms of schizophrenia were poorly delineated, but to eliminate in spite of improving them or prompting further research, is an arbitrary decision taken by specialists that follows a school of psychopathology were imprecision seems to be the constitutive. Scarce evidence against the existence of schizophrenia subclinical forms was selected to make such a decision (Tandon et al., 2013) [2]. And this was the thread of thinking for DSM5 Schizophrenia chapter. We need to remember the fact that DSM successive editions, were mostly prompted by the need of giving momentum to clinical diagnosis in a context where a poor descriptive psychopathology tradition was the rule, in favour of a dynamic approach to clinical problems, where diagnosis were almost forbidden (Kandel, 1998) [3].

Since Bleuler's "Dementia Praecoxand the Group of Schizophrenias (Bleuler, 1950)" first publishing, phenotypic heterogeneity of schizophrenias has been a clinical assumption with clear descriptions made by well renowned experts. Hebephrenias are quite good delineated in Haecker's and Bleuler reports. Paranoid
Marcelo Cetkovich Bakmas ${ }^{123}$

1 Department of Psychiatry, Institute of Cognitive Neurology (INECO), Pacheco de Melo 1854 (1126) Buenos Aires, Argentina

2 Department of Psychiatry, Institute of Neurosciences, Favaloro Foundation Univeristy Hospital, Argentina

3 Department of Mental Health and Psychiatry, School of Medicine, Favaloro Foundation University, Argentina

Corresponding author: Marcelo Cetkovich Bakmas

” mcetkovich@ineco.org.ar, mcetkovich@ffavaloro.org

Director, Department of Psychiatry, Institute of Cognitive Neurology (INECO), Pacheco de Melo 1854 (1126) Buenos Aires, Argentina.

Tel: (54-1-1) 4781-7684

Citation: Bakmas MC. Differentiated Psychopathology and Molecular Genetics of Endogenous Psychosis: Much More than a Misunderstanding. Acta Psychopathol. 2015, 1:3.

schizophrenias, as well as paraphrenias were also clearly described by Kraepelin. First catatonic's states observed by Kahlbaum were, mostly, affective or even epileptic acute states (Jahn \& Thomas, 2004) [4]. What Kraepelin did, was to realize that all this different clinical pictures had something in common, and put all them together and coin the term "Dementia Praecox", separating them from affective Manic-Depressive Illness (Kraepelin \& Emil, 1904) [5]. In his late writings Kraepelin realized that Dementia Praecox may comprise several diseases. Later on, the so called WernickeKleist-Leonhard school of Psychiatry, made the first attempt to gather all endogenous psychosis in one classification that, for the first time, took in account not only clinical presentations, but also modes of onset, outcome, prognosis and family genetic loading. It worth's a close reading of Leonhard's book, "The classification of Endogenous Psychosis and Their Differentiated Psychopathology" (now available in a new English version edited by the Late Helmut Beckmann (Leonhard, 2012)) [6]. It shows that, for example, Leonhard was the very first one that, based on his own 
statistical data, posted back in the sixties, that environmental factors must be involved in schizophrenia etiology; this was a shocking idea at that time, but strong evidence support this approach nowadays (Van Os et al., 2010) [7]. Looking at Leonhard's classification we can see that, being a categorical classification assuming the existence of different disease entities, it is mostly based on dimensional traits.

Karl Leonhard's rationale for splitting endogenous psychoses, particularly schizophrenia is parsimonious: if we can describe conditions with different clinical traits, onset, outcome and family loading, in spite of not having yet the biological explanation, it worth's to keep them separated waiting for research to give an answer. He takes the example of neurologists that keep apart clinical forms of the same disease only taking in account factors like age of onset or family history, no matter the clinical picture being the same. (Leonhard's Book Introduction)

Kleist and Leonhard, studying affective psychosis, postulated the existence of "unipolar" and "bipolar" disorders, a concept broadly accepted today (Marneros et al., 2002) [8]. Besides affective disorders, Leonhard describes three main group of psychosis: Cycloid Psychosis (acute onset, good prognosis, low family loading, not belonging to the "bipolar spectrum") (Pfuhlmann et al., 2004) [9], and the schizophrenias that he splits in two groups: Unsystematic and Systematic ones, (because what he thought was the affectation of one or several "brain systems"). Unsystematic schizophrenias are characterized by acute polymorphous episodes with variable defect states, depending on the number of episodes. This group have a high family loading, being Periodic Catatonia the example of a family disease (Schanze et al., 2012) [10]. Systematic schizophrenias are characterized by an insidious onset, with highly nonspecific early symptoms and progression to states of high functional impairment. This group represent a sporadic form of schizophrenias with almost no family loading. Leonhard's as well as others data, in spite of being of low power; confirm this different genetic loading in both groups of schizophrenias. Franzek and Beckmann twin study confirmed a high family loading in unsystematic schizophrenias, with almost no heredity traits in unsystematic (Franzek \& Beckmann, 1998) (Pfuhlmann et al., 1999) [11, 12]. For this school of research, Unsystematic schizophrenias have a higher genetic loading than systematic schizophrenias (Beckmann \& Franzek, 2000) [13]. An interesting approach from Leonhard was the interplay between what he termed "pathogenic" and "pathoplastic" factors. Pathogenetic would be all factors that confers a general risk for the development of disease, this includes genetic and developmental factor in nowadays knowledge. Pathoplastic refers to all factors that contribute to give each clinical form, its particular profile. All this explain why Leonhard's includes "differentiated etiology" on the title of his book. This is an important aspect that is not properly taken in account by the modern "Genes by Environment" model (Van Os et al., 2010) [7]: the possibility that genetic and environmental factors are not distributed normally and that within the "group of schizophrenias" there are cases where genetic loading is most determinant and other were environmental factors prevail. It was argued that this classification is hard to learn, but it only needs a proper training (Pfuhlmann et al., 1997) [14].
Dimensional approach has been the mainstream in searching for the causes of endogenous psychosis during last years (Jablensky \& Assen, 2005) [15]; here we would like to highlight recent research that gives support to the opposite idea, i.e.; that between the names of schizophrenia several diseases could be included, as Leonhard and others postulated decades ago.

Kenneth Kendler is one of the more lucid researchers in the field of genetic of schizophrenia. In a recent a thoughtful comprehensive paper he grouped predictive variables in psychiatric research in three groups: psychological (that includes symptoms), biological (including genetic, molecular and structural) and environmental (including individual, familial and social) (Kenneth $\mathrm{S}$ Kendler, 2014) [16]. Kendler please that researchers must bear in mind this schemata, in order to ascribe their data to one of this levels of research, for the seek of order. Being a supporter of a dimensional approach he has also carried out studies with data showing that psychosis involves several phenotypes, and not only two diseases, like in the Roscommon study (KS Kendler et al., 1998; KS Kendler et al., 1995) [17, 18]. In a recent comprehensive review Kendler highlights the difficulties of genetic research in psychiatry that clearly explain methodological differences between dimensional and categorical approaches, and explain how they are rooted in two different genetic research traditions, "biometricians" and "Mendelians" (KS Kendler, 2014) [19]. In this paper Kendler concludes that "the historical effort to ground the categorical nature of schizophrenia has failed". This assumption is based mostly in Genome Wide Scan Studies (GWAS), where imprecise clinical profiling of phenotypes under study is almost the rule. Most of this studies struggle to find genetic markers of schizophrenia as an absent-present phenomena, bypassing most subtle clinical traits. Some researchers based on this results argue that, even splitting schizophrenia from bipolar disorders could be an artifact and that boundaries are not clear (Owen et al., 2007) (Craddock et al., 2009) [20, 21]. Our point of view is that these looks like a "post hoc propter hoc" interpretation of results.

A recent and highly debated paper published by Arnedo et al shows strong evidence that such an affirmation could be wrong (Arnedo et al., 2015) [22]. Through a complex statistical analysis of data from three different GWAS studies they show a complex molecular and clinical architecture combination demonstrating the categorical clinical heterogeneity of schizophrenia. They started pointing those complex diseases like schizophrenia could be affected by hundred thousands of genetic variables interacting between them showing a complex genetic architecture. Multiple and different paths of interaction leads to multiple aspects of the disease. The idea of "one gene one disease" must be abandoned. Second, they refer that the architecture of genetic of heritable diseases refers to the number, frequency and size of the effect of genetic risk alleles and the way they are organized in genotypic networks. In complex disorders, same genotypic networks could lead to different clinical outcome (multifinality or genetic pleiotropy) and different genetic networks could lead to the same clinical outcome (equifinality or heterogeneity). Geneticist must expect that several genes affecting each trait and each gene affecting several traits. For Arnedo et al this simple genetic research rules, make understandable that heritable disorder's research have 
the chance to lead to weak and inconsistent results, unless their complex genetic and phenotypic complexity is taken in account. This is the key aspect of their research, the very sophisticated combination of two complexities. They lost the assumption of schizophrenia being a single variable: absent or present, and deal with the idea of a complex phenotypic architecture and went for it. They took clinical data from three different GWAS studies and selected 93 clinical traits from the "Diagnostic Interview for Clinical Studies". They used a generalized factorization method combined with non-negative matrix factorization, in order to identify candidates for functional clusters. In this way they were able to identify 343 different phenotypic groups with chance of overlap, with different clinical characteristics grouped in particular cases of schizophrenia. Moreover: phenotypic groups of clinical syndromes, in an independent way of their genetic base. They also used the same method to find several interacting sets of single nucleotide polymorphisms that clustered in certain individuals, regardless their clinical status. Then, they tested whether SNPs sets were associated with distinct phenotypic sets, and they allow the researchers to find "eight classes of schizophrenias" according a particular combination of clinical profile and SNPS sets. Arnedo et al conclude that their findings indicate that schizophrenia comprise several different clinical syndromes associated with several genotypic networks disassembled. Then, most of heritability of schizophrenia has been not detected when the paradigm present-absent is used. Their purely data driven analysis shows that elusive heritability of schizophrenia is not lost, but encrypted within a complex distribution of relationships between genotypes and phenotypes. They admit that their evidence indicating that schizophrenia is a heterogeneous group of diseases suggest that reduction of clinical information about schizophrenia to a single categorical diagnosis as inadequate.

Shortly after this paper, another one very similar demonstrated a complex phenotype-genotype interaction in the field of Affective disorders (Xu et al., 2015) [23]. Using clinical data from the General Health Questionnaire (GHQ) which have four dimensions, Xu et al were able to find a stronger association sub phenotypes with SNPs with a high size effect. We find in this paper another example of how a better definition of clinical phenotypes, this time extracted from a short questionnaire, clinical-genetic correlation could be improved.

In conclusion: modern mathematical approach to the highly complex issue of the "architecture" of mental disorders ethology, particularly genetics, shows that phenotypes are far more complex than mostly assumed by current diagnostic manuals. For this, a psychopathological dimensional approach has been proposed as an alternative. On the other hand, reviewed studies like Arnedo's revisit the classic categorical approach in psychiatry: that mental disorders can be subdivided, according clinical traits, in several sub forms like, for example, the Wernicke Kleist Leonhard School of Psychiatry proposed decades ago.

During the last meeting of the International Society of Bipolar Disorder in Toronto Heinz Grunze gave a lecture with the suggestive title of "Apples and Peas are similar but not the same". After reviewing all clinical differences demonstrating why schizophrenia and bipolar disorder are different conditions he concluded: "The less scientific argument but the more relevant from a clinical point of view: an experimented clinician can say immediately who is who". And he sums up the problem saying that "finally, all brain diseases meet in some point of continuity of affectation of the same organ, having the same neurotoxic paths, their phenotypes could overlap in a significative way". We propose that the same principles could be applied to clinical sub forms of schizophrenia.

Following times in Neuroscience research of schizophrenia and other conditions like bipolar disorder, will witness renewal of interest in psychopathology. Explanation and understanding, as proposed by Karl Jaspers one hundred year ago (Stanghellini \& Fuchs, 2013) [24], are the two main tools of descriptive psychopathology, the cognitive system that allow us to get access to our patients suffering and complains (Berrios, 1996) [25]. Empathy, core feature of psychopathology, became focus of complex neurobiological research, also (Melloni et al., 2014) [26].

This framework push us to remember the old paragraph: "Looking for news? read the classics". 


\section{References}

1 American Psychiatric Association (2013) Diagnostic and Statistical Manual of Mental Disorders (DSM- ${ }^{\circledR}$ ). American Psychiatric Pub.

2 Tandon R, Gaebel W, Barch DM, Bustillo J, Gur RE, et al. (2013) Definition and description of schizophrenia in the DSM-5. Schizophrenia Research 150: 3-10.

3 Kandel ER (1998) A New Intellectual Framework for Psychiatry. The American Journal of Psychiatry 155: 457-469.

4 Jahn T \& Thomas J (2004) Katatonie-130 Jahre nach Karl Ludwig Kahlbaum. In Bewegungsstörungen bei Psychischen Erkrankungen 3-27.

5 Kraepelin E \& Emil K (1904) Lectures on clinical psychiatry.

6 Leonhard K (2012) Classification of Endogenous Psychoses and their Differentiated Etiology. Springer Science \& Business Media.

7 Van Os J, Kenis G, Rutten BPF (2010) The environment and schizophrenia. Nature 468: 203-212.

8 Marneros A, Andreas M, Jules A (2002) Bipolar disorders: roots and evolution. In Bipolar Disorders 1-35.

9 Pfuhlmann B, Jabs B, Althaus G, Schmidtke A, Bartsch A, et al. (2004) Cycloid psychoses are not part of a bipolar affective spectrum: results of a controlled family study. Journal of Affective Disorders 83: 11-19.

10 Schanze D, Ekici AB, Pfuhlmann B, Reis A, Stober G (2012) Evaluation of conserved and ultra-conserved non-genic sequences in chromosome 15q15-linked periodic catatonia. American Journal of Medical Genetics. Part B, Neuropsychiatric Genetics: The Official Publication of the International Society of Psychiatric Genetics 159: 77-86.

11 Franzek E \& Beckmann H (1998) Different genetic background of schizophrenia spectrum psychoses: a twin study. The American Journal of Psychiatry 155: 76-83.

12 Pfuhlmann B, Franzek E, Beckmann H (1999) Absence of a subgroup of chronic schizophrenia in monozygotic twins. Consequences for considerations on the pathogenesis of schizophrenic psychoses. European Archives of Psychiatry and Clinical Neuroscience 249: 50-54.

13 Beckmann H \& Franzek E (2000) The genetic heterogeneity of "schizophrenia." The World Journal of Biological Psychiatry: The Official Journal of the World Federation of Societies of Biological Psychiatry 1: 35-41.
14 Pfuhlmann B, Franzek E, Stober G, Cetkovich-Bakmas M, Beckmann H (1997) On interrater reliability for Leonhard's classification of endogenous psychoses. Psychopathology 30: 100-105.

15 Jablensky A \& Assen J (2005) Categories, Dimensions and Prototypes: Critical Issues for Psychiatric Classification. Psychopathology 38: 201-205.

16 Kendler KS (2014) A joint history of the nature of genetic variation and the nature of schizophrenia. Molecular Psychiatry 20: 77-83.

17 Kendler KS, Karkowski LM, Walsh D (1998) The structure of psychosis: latent class analysis of probands from the Roscommon Family Study. Archives of General Psychiatry 55: 492-499.

18 Kendler KS, Neale MC, Walsh D (1995) Evaluating the spectrum concept of schizophrenia in the Roscommon Family Study. The American Journal of Psychiatry 152: 749-754.

19 Kendler KS (2014) The Structure of Psychiatric Science. The American Journal of Psychiatry 171: 931-938.

20 Owen MJ, Craddock N, Jablensky A (2007) The genetic deconstruction of psychosis. Schizophrenia Bulletin 33: 905-911.

21 Craddock N, O'Donovan MC, Owen MJ (2009) Psychosis genetics: modeling the relationship between schizophrenia, bipolar disorder, and mixed (or "schizoaffective") psychoses. Schizophrenia Bulletin 35: 482-490.

22 Arnedo J, Javier A, Svrakic DM, del Val C, Rocío RZ, et al. (2015) Molecular Genetics of Schizophrenia Consortium. Uncovering the Hidden Risk Architecture of the Schizophrenias: Confirmation in Three Independent Genome-Wide Association Studies. The American Journal of Psychiatry 172: 139-153.

23 Xu MK, Gaysina D, Barnett JH, Scoriels L, van de Lagemaat LN, et al. (2015) LHA Genetics Group. Psychometric precision in phenotype definition is a useful step in molecular genetic investigation of psychiatric disorders. Translational Psychiatry 5: e593.

24 Stanghellini G \& Fuchs T (2013) One Century of Karl Jaspers General Psychopathology. OUP Oxford.

25 Berrios GE (1996) Descriptive psychopathology. In Descriptive Psychopathology since the Nineteenth Century 15-32.

26 Melloni M, Margherita M, Vladimir L, Agustin I (2014) Empathy and contextual social cognition. Cognitive, Affective \& Behavioral Neuroscience 14: 407-425. 